\title{
MDMA Impairs Both the Encoding and Retrieval of Emotional Recollections
}

\author{
Manoj K Doss", , Jessica Weafer ${ }^{2}$, David A Gallo' and Harriet de Wit ${ }^{2}$ \\ 'Department of Psychology, University of Chicago, Chicago, IL, USA; ${ }^{2}$ Department of Psychiatry and Behavioral Neuroscience, University of Chicago, \\ Chicago, IL, USA
}

\begin{abstract}
The psychoactive drug \pm 3 ,4-methylenedioxymethamphetamine (MDMA) is increasingly used for its perceived emotional effects (eg, prosociality, empathy, psychotherapy), but surprisingly little research has been aimed at identifying the effect of the drug on emotional episodic memory in humans. Here, we report the first double-blind placebo-controlled study to examine the effects of MDMA on emotional memory separately during encoding and retrieval in healthy participants. Participants viewed emotionally negative, neutral, and positive pictures and their labels. Forty-eight hours later, they were given cued recollection and recognition memory tests designed to assess recollection and familiarity for the studied pictures. Participants were randomly assigned to one of three groups who received MDMA (I mg/kg) either during encoding (Encoding group; $N=20$ ), retrieval (Retrieval group; $N=20$ ), or neither (Placebo group; $N=20$ ). Although MDMA administered at either phase did not affect overall memory accuracy, it did alter the recollection of details associated specifically with emotional memories as estimated using a dual process signal detection analysis of confidence judgments and subjective 'remember' judgments. In the Encoding group, MDMA reduced recollection estimates for negative and positive pictures but had little to no effect on neutral items or familiarity estimates. There was evidence for similar trends in the Retrieval group. These findings indicate that MDMA attenuates the encoding and retrieval of salient details from emotional events, consistent with the idea that its potential therapeutic effects for treating posttraumatic stress disorder are related to altering emotional memory.

Neuropsychopharmacology (2018) 43, 79I-800; doi:I0.1038/npp.2017.171; published online 27 September 2017
\end{abstract}

\section{INTRODUCTION}

Recent findings from clinical and basic research suggests that the stimulant-psychedelic-entactogen $\pm 3,4$-methylenedioxymethamphetamine (MDMA) may specifically modulate emotional processing in humans. MDMA has reemerged as a potential treatment of posttraumatic stress disorder (PTSD; Sessa, 2016) possibly due to its prosocial effects (Bershad et al, 2016), which may make it easier for patients to discuss traumatic events. Alternatively, MDMA may modify traumatic memories themselves, consistent with the characterization of PTSD as a disorder of emotional memory (Rubin et al, 2008). Patients with PTSD exhibit hypervigilance to threat and a hyperresponsive amygdala (Rauch et al, 2006) while in neurotypical populations, MDMA attenuates the detection of threat-related emotional faces (Hysek et al, 2014) and amygdalar activation (Bedi et al, 2009). Together, such findings imply that MDMA may alter emotional memory.

To date, there have been no studies on how MDMA might affect emotional episodic memory, but there is some evidence that MDMA impairs memory for emotionally

\footnotetext{
*Correspondence: MK Doss, Department of Psychology, The University of Chicago, 5848 S. University Ave., Beecher 308, Chicago, IL 60637, USA. Tel: + I 773702 4376, E-mail: mkdoss@uchicago.edu

Received 8 May 2017; revised 19 July 2017; accepted 6 August 2017; accepted article preview online 21 August 2017
}

neutral information. MDMA worsens memory when it is administered before the encoding of neutral verbal stimuli (Kuypers and Ramaekers, 2005), and this effect can be reversed by blocking the $5-\mathrm{HT}_{2 \mathrm{~A}}$ receptor (van Wel et al, 2011), a site that binds psychedelic drugs and differentiates MDMA from classic stimulants. However, these studies tested memory shortly after encoding while participants were still intoxicated, making it difficult to determine whether MDMA affected encoding or retrieval.

Although prior work with MDMA has revealed a potentially amnestic effect on emotionally neutral memories, MDMA also has a stimulating effect, suggesting that its profile for emotional episodic memories may be more complex. Specifically, we have found that classic stimulants (eg, dextroamphetamine) have greater specificity for enhancing memories with emotional content (Ballard et al, 2013). Both dextroamphetamine and MDMA drive the noradrenergic system, which is thought to support emotional memory enhancements (Mather et al, 2016). It may be that any amnestic effects of MDMA on emotional memory are negated by its effects on the noradrenergic system.

MDMA may also alter memory during retrieval, independently of its potential encoding effects. Although the effects of MDMA on episodic memory retrieval have not been tested, there is evidence that MDMA enhances subjective vividness and positivity ratings of positive autobiographical memories while reducing negativity ratings of negative 
autobiographical memories (Carhart-Harris et al, 2014). In this way, MDMA may have a similar effect on retrieval as dextroamphetamine, which increases memory errors and recall of positively valenced words (Ballard et al, 2014). However, there may again be differences between MDMA and classic stimulants. In mice, MDMA reduces freezing when a fear memory is recalled during extinction (Young et al, 2015), whereas dextroamphetamine does not reduce conditioned freezing during fear extinction (Carmack et al, 2010). These findings suggest that MDMA may have specific impact on the retrieval of emotional memory representations.

The present study sought to disentangle the effects of MDMA on the encoding and retrieval of negative, neutral, and positive memory. Additionally, we sought to distinguish the MDMA effects on two components of episodic memory retrieval: recollection and familiarity. Recollection is characterized by the retrieval of specific details associated with a prior event and is known to be hippocampally-dependent, whereas familiarity is the feeling of knowing an event has occurred without the recollection of specific details and is thought to be cortically-dependent (Yonelinas, 2002). PTSD patients exhibit abnormal hippocampal structure (Smith, 2005) and function (Brohawn et al, 2010) and possess vivid recollections of traumatic events. These observations, combined with the fact that emotion specifically enhances recollection (Phelps and Sharot, 2008), suggest that the clinical efficacy of MDMA may be related to its effects on emotional recollection. Therefore, we predicted that MDMA would attenuate both the encoding and retrieval of recollection-based emotional memory.

\section{MATERIALS AND METHODS}

\section{Participants}

Sixty healthy participants (20 per group, 50\% males, $18-34$ years) were recruited. Potential participants underwent a physical examination and an electrocardiogram provided detailed information on current and lifetime drug use, and were screened by trained clinical psychologists using a semistructured psychiatric interview on average 17 weeks before the first experimental session. Exclusion criteria included current Axis I DSM-IV disorder, including substance dependence, $>5$ cigarettes per day, history of psychosis or mania, less than a high school education, lack of English fluency, a body mass index outside $19-30 \mathrm{~kg} / \mathrm{m}^{2}$, high blood pressure $(>140 / 90)$, abnormal electrocardiogram, daily use of any medication other than birth control, pregnancy, or lactating. Participants were eligible if they reported 4-40 past uses of MDMA with no adverse events. Women not taking hormonal contraceptives were tested during their follicular phase because hormonal fluctuations can influence responses to stimulants (White et al, 2002). There were no group differences in participant demographics (Table 1).

Qualifying participants attended an orientation session to give consent and practice study tasks. To minimize expectancy, participants were informed that they could receive a stimulant, sedative, cannabinoid, or placebo. Participants were instructed to consume their normal amounts of caffeine and nicotine before sessions but to
Table I Demographic Data of Groups

\begin{tabular}{|c|c|c|c|}
\hline & Placebo & $\begin{array}{l}\text { MDMA at } \\
\text { encoding }\end{array}$ & $\begin{array}{l}\text { MDMA at } \\
\text { retrieval }\end{array}$ \\
\hline Female/male & $10 / 10$ & $10 / 10$ & $10 / 10$ \\
\hline Age (years) & $23.85(0.34)$ & $24.90(0.21)$ & $22.45(0.10)$ \\
\hline Education (years) & $15.10(0.11)$ & I $5.3(0.09)$ & | $4.85(0.08)$ \\
\hline BMI & $22.62(0.11)$ & $24.37(0.16)$ & $23.32(0.13)$ \\
\hline \multicolumn{4}{|l|}{ Race } \\
\hline Caucasian & $45 \%$ & $55 \%$ & $55 \%$ \\
\hline Black & $20 \%$ & $5 \%$ & $5 \%$ \\
\hline Asian & $5 \%$ & $25 \%$ & $15 \%$ \\
\hline $\begin{array}{l}\text { Other (includes } \\
\text { multiracial) }\end{array}$ & $30 \%$ & $15 \%$ & $25 \%$ \\
\hline \multicolumn{4}{|l|}{ Current drug use } \\
\hline $\begin{array}{l}\text { Caffeinated drinks } \\
\text { per day }\end{array}$ & $1.67(0.05)$ & $1.04(0.04)$ & $1.24(0.06)$ \\
\hline Cigarettes per day & $1.14(0.10)$ & $0.69(0.06)$ & $0.74(0.08)$ \\
\hline \% Smokers & $55 \%$ & $40 \%$ & $50 \%$ \\
\hline $\begin{array}{l}\text { Alcoholic drinks per } \\
\text { week }\end{array}$ & $4.13(0.11)$ & $3.80(0.09)$ & $3.80(0.12)$ \\
\hline \multicolumn{4}{|c|}{ Lifetime drug use (at least once) } \\
\hline Marijuana & $100 \%$ & $100 \%$ & $100 \%$ \\
\hline Sedatives & $65 \%$ & $65 \%$ & $50 \%$ \\
\hline Stimulants & $100 \%$ & $90 \%$ & $80 \%$ \\
\hline Opiates & $80 \%$ & $85 \%$ & $75 \%$ \\
\hline Hallucinogens & $80 \%$ & $95 \%$ & $85 \%$ \\
\hline $\begin{array}{l}\text { Lifetime uses of } \\
\text { MDMA }\end{array}$ & I I.50 (0.4 I) & I5.95 (0.47) & $10.98(0.4 \mid)$ \\
\hline $\begin{array}{l}\text { Last use of MDMA (in } \\
\text { years) }\end{array}$ & $1.26(0.40)$ & $1.05(0.35)$ & $0.80(0.20)$ \\
\hline
\end{tabular}

Non-percent values are mean and SEM in parentheses. Lifetime drug use refers to recreational use only.

abstain from using alcohol, prescription drugs (except contraceptives), and over-the-counter drugs for $24 \mathrm{~h}$ before the sessions, marijuana for $72 \mathrm{~h}$ before the sessions, and other illicit drugs for $48 \mathrm{~h}$ before the sessions (due to faster clearance). Participants were notified that there would be drug tests and that they would be rescheduled if they tested positive for any recent drug use at the first session and cancelled from the study if they tested positive at the second session with only partial compensation. Participants were advised to get their normal amounts of sleep and not to eat for $2 \mathrm{~h}$ before each session. Following completion of the study, participants were fully debriefed and monetarily compensated. The study took place at the University of Chicago Medical Center and was approved by the Institutional Review Board.

\section{Drug}

MDMA ( $1.0 \mathrm{mg} / \mathrm{kg})$ was prepared for each participant by the hospital pharmacist. The powder form of the drug was 
obtained from Dr David Nichols of Purdue University and placed in opaque size 00 capsules with dextrose filler. Placebo capsules contained only dextrose. This is a moderate dose of MDMA relative to doses that previously affected memory (75 mg in Kuypers and Ramaekers, 2005).

\section{Design}

Subjects were randomly assigned to one of three groups, one that received MDMA during encoding and placebo during retrieval (Encoding), one that received MDMA during retrieval and placebo during encoding (Retrieval), and one that received placebo during both phases (Placebo). All participants attended two sessions separated by $48 \mathrm{~h}$ : an encoding session for studying stimuli and a retrieval session for testing memory. Besides the drug manipulation, the procedure for all groups was identical and double-blinded. All sessions began in the morning.

\section{Stimuli}

Stimuli consisted of 180 images from the International Affective Picture Set (IAPS; Lang et al, 2008) and 2-3 word labels (eg, 'dirty toilet', 'box of tissues', 'chocolate candy bar') describing these images. The images included emotionally negative, neutral, and positive pictures and were split into two comparable sets for counterbalancing studied and nonstudied items across participants. These pictures had the following mean (SD) normed valences and arousals, respectively: Set A: negative $3.09(0.51)$ and $5.21(0.66)$, neutral $5.15(0.43)$ and $3.51(0.68)$, positive $7.10(0.52)$ and 5.00 (0.79). Set B: negative $3.14(0.50)$ and $5.21(0.65)$, neutral $5.10(0.55)$ and $4.12(0.90)$, positive $7.08(0.53)$ and 5.17 (0.77).

\section{Procedure}

On the morning of experimental sessions, participants first completed compliance measures including breath alcohol level (Alco-sensor III; Intoximeters, St Louis, MO), a urine drug test (ToxCup, Branan Medical, Irvine, CA), and a pregnancy test (females only; Aimstrip, Craig Medical, Vista, CA), as well as baseline cardiovascular and mood measures. Participants then consumed a capsule and completed cardiovascular and mood measures every $30 \mathrm{~min}$ for the next $90 \mathrm{~min}$. Participants were provided with magazines and music in furnished rooms. They were not allowed to eat, sleep, or work, and they had no access to cell phones or Internet. Upon completing tasks, participants watched a movie.

During the encoding session, $90 \mathrm{~min}$ post-capsule ingestion, participants viewed all 180 labels, half of which were followed by the corresponding picture. For each label, participants rated on a 5-point scale how much they would like to see the corresponding picture. When a picture was presented, participants rated its positivity and negativity on a $5 \times 5$ grid with positivity and negativity on orthogonal axes. After this valence rating, they rated the picture's arousal on a five-point scale. This phase was self-paced and lasted approximately $30 \mathrm{~min}$. There were no group differences in liking ratings of labels and valence/arousal ratings of images, so these will not be reported.
During the retrieval session, 90 min post-capsule ingestion, participants were given two surprise memory tests, a cued recollection test and a picture recognition test. For the cued recollection test, participants were presented with each label and asked whether they had seen the corresponding picture. Afterward, they rated their confidence on a five-point scale and were encouraged to use the entire scale. After the cued recollection test, participants were presented with each picture and had to decide if it had been seen. When a picture was recognized, they were asked if they 'remember' the picture or they simply 'know' it was presented (Yonelinas, 2002). Participants were instructed that they should give a 'remember' response when they could recollect associated details from the event, such as thoughts during its presentation, and they should give a 'know' response when they simply knew that a picture had been presented without recollecting specific details. Both memory tests were selfpaced and together lasted approximately $45 \mathrm{~min}$.

\section{Dependent Measures}

Several measures were obtained to monitor expected drug effects (Table 2). Heart rate and blood pressure were measured using a portable blood pressure monitor (A\&D Medical/Life Source, San Jose, CA). Mood measures included the Profile of Mood States (McNair et al, 1971), the Visual Analog Scales (Folstein and Luria, 1973), the Drug Effects Questionnaire (Morean et al, 2013), and an End of Session Questionnaire. See Supplementary Online Materials (SOM) for descriptions of each scale and statistics.

For the cued recollection test (Table 3), hit and false alarm rates were calculated for each valence in each subject. False alarms were subtracted from hits to compute memory accuracy. Finally, high confidence hits, false alarms, and accuracy were calculated by only including responses with the top two levels of confidence (SOM).

To estimate recollection and familiarity, confidence data were submitted to a dual process signal detection (DPSD) analysis (Yonelinas, 2002) using the ROC Toolbox for MATLAB (Koen et al, 2016). Confidence data were combined between 'yes' and 'no' responses to create a 10-point scale. The cumulative proportion of hits is plotted against the cumulative proportion of false alarms from the most stringent criterion (ie, the proportion of hits and false alarms at the highest level of confidence) to the most liberal criterion, ending at $(1,1)$. A receiver operator characteristic (ROC) curve is then fit to these points using maximum likelihood estimation. The DPSD model assumes a threshold process (recollection) can take place on some proportion of trials that is reflected by the $y$ intercept (measured as a probability). In contrast, familiarity is thought to be a signal detection process, reflected in the curvilinearity of the function (measured in $z$ score units).

For the recognition test (Table 4), hits, false alarms, and accuracy were calculated, and recollection and familiarity estimates were derived from the independence remember/ know (IRK) procedure (Yonelinas, 2002). Recollection accuracy was measured by

$$
p(\text { "remember"|old })-p(\text { "remember"|new })
$$

Because a 'know' response is the probability of familiarity in the absence of recollection, a correction was made to avoid 
Table 2 Physiological and Mood Measures

\begin{tabular}{|c|c|c|c|c|c|c|}
\hline & \multicolumn{3}{|c|}{ Session I } & \multicolumn{3}{|c|}{ Session 2} \\
\hline & Placebo & MDMA at encoding & MDMA at retrieval & Placebo & MDMA at encoding & MDMA at retrieva \\
\hline Heart rate & $-7.70(1.09)$ & $6.20(1.87)$ & $-3.20(1.98)$ & $-3.80(1.67)$ & $-9.60(1.65)$ & $6.35(1.78)$ \\
\hline Systolic BP & $-3.55(2.32)$ & $16.55(2.17)$ & $0.15(2.02)$ & $-1.95(1.48)$ & $-2.75(1.90)$ & $12.65(6.10)$ \\
\hline Diastolic BP & $-1.15(2.76)$ & $8.85(2.07)$ & $0.85(2.22)$ & $-3.00(1.23)$ & $-1.30(1.95)$ & I I.80 (2.21) \\
\hline \multicolumn{7}{|l|}{ POMS } \\
\hline Friendliness & $-2.45(0.61)$ & $-1.35(1.57)$ & $-4.40(1.00)$ & $-1.95(0.63)$ & $-2.30(0.92)$ & $0.60(1.07)$ \\
\hline Anxiety & $-0.10(0.51)$ & $2.15(0.92)$ & $-0.50(0.77)$ & $0.65(0.39)$ & $0.05(0.15)$ & $6.85(1.81)$ \\
\hline Elation & $-1.40(0.61)$ & $-0.30(1.26)$ & $-2.00(0.62)$ & $-1.50(0.67)$ & $-1.35(2.43)$ & $2.25(0.88)$ \\
\hline Anger & $-0.60(0.36)$ & $-0.1(0.42)$ & $-2.45(0.86)$ & $-0.40(0.17)$ & $0.05(0.22)$ & $1.25(0.79)$ \\
\hline Fatigue & $0.65(0.74)$ & $0.45(1.21)$ & $-0.15(0.73)$ & $0.30(0.46)$ & $0.8(0.49)$ & $-0.25(0.64)$ \\
\hline Depression & $-0.55(0.5 I)$ & $1.10(0.72)$ & $-1.90(1.09)$ & $-0.30(0.33)$ & $0.05(0.22)$ & $2.20(1.37)$ \\
\hline Confusion & $0.10(0.52)$ & $2.30(0.75)$ & $-0.25(0.63)$ & $0.25(0.26)$ & $0.1(0.42)$ & $2.85(1.04)$ \\
\hline Vigor & $-3.10(0.8 I)$ & $0.00(2.14)$ & $-4.30(1.20)$ & $-1.85(1.11)$ & $-2.60(0.75)$ & $3.60(1.85)$ \\
\hline \multicolumn{7}{|l|}{ VAS } \\
\hline Anxious & $-1.35(1.63)$ & $8.40(5.14)$ & $-2.00(4.35)$ & $-1.10(2.68)$ & $-2.25(1.44)$ & 25.80 \\
\hline Stimulated & $2.40(4.62)$ & $27.10(7.01)$ & $-1.90(4.45)$ & $0.35(4.01)$ & $-2.85(1.93)$ & $40.60(6.36)$ \\
\hline Sedated & | $8.40(6.21)$ & I $6.00(7.47)$ & $12.25(4.76)$ & $6.75(6.03)$ & $9.60(3.19)$ & $-0.10(5.17)$ \\
\hline Elated & $0.40(3.79)$ & I I.80 (6.05) & $-4.70(3.48)$ & $1.85(3.61)$ & $-0.85(1.47)$ & $16.80(5.62)$ \\
\hline Insightful & $0.05(4.44)$ & I $2.25(4.00)$ & $-2.45(4.29)$ & $0.45(2.33)$ & $1.90(2.85)$ & $28.80(5.05)$ \\
\hline Sociable & $-4.60(4.38)$ & $2.10(5.09)$ & $-5.60(4.49)$ & $-2.20(3.07)$ & $-2.00(3.86)$ & $15.00(6.94)$ \\
\hline Confident & $-6.05(2.18)$ & $-7.10(4.83)$ & $-5.15(3.20)$ & $-0.75(2.89)$ & $-1.15(4.21)$ & $13.15(7.84)$ \\
\hline Lonely & $4.90(4.35)$ & $9.60(4.67)$ & $-1.30(3.34)$ & $0.95(1.75)$ & $4.60(2.91)$ & $12.30(7.10)$ \\
\hline Playful & $-7.65(4.56)$ & $2.50(5.69)$ & $-0.95(4.30)$ & 3.15 (3.39) & $-3.30(2.83)$ & $17.25(7.92)$ \\
\hline Dizzy & $2.75(3.14)$ & $6.30(4.93)$ & $-1.65(1.14)$ & $5.90(3.64)$ & $-0.05(1.99)$ & I7.60 (5.30) \\
\hline Loving & $-11.10(4.87)$ & $13.40(5.13)$ & $-0.60(4.33)$ & $-2.70(2.10)$ & $-1.20(2.77)$ & $16.20(5.52)$ \\
\hline Friendly & $-8.95(2.08)$ & $2.70(4.33)$ & $-9.55(4.06)$ & $-1.10(2.79)$ & $-5.35(3.12)$ & $7.80(6.98)$ \\
\hline Restless & $5.60(3.61)$ & I5.25 (6.1 I) & $10.00(3.77)$ & $4.60(4.04)$ & $11.00(5.18)$ & $31.85(8.57)$ \\
\hline \multicolumn{7}{|l|}{ DEQ } \\
\hline Feel drug effect & $21.50(4.70)$ & $50.60(5.60)$ & I 1.30 (3.69) & I4. 15 (5.02) & $6.40(2.52)$ & $58.75(5.75)$ \\
\hline Like drug effect & $34.40(7.56)$ & $57.20(7.24)$ & $21.45(6.94)$ & | 8.05 (5.45) & $8.95(3.94)$ & $62.55(6.57)$ \\
\hline Dislike drug effect & $7.60(2.47)$ & I $9.25(4.58)$ & $3.70(1.60)$ & $7.95(2.88)$ & $2.90(1.16)$ & $21.40(5.41)$ \\
\hline Feel high & I7.40 (4.5I) & $48.45(6.96)$ & $10.95(4.06)$ & I I.50 (4.83) & $6.95(3.03)$ & $53.25(6.46)$ \\
\hline Want more drug & $52.20(8.07)$ & $44.75(7.53)$ & $36.85(8.45)$ & $27.65(5.89)$ & | $4.85(6.46)$ & $48.80(8.08)$ \\
\hline \multicolumn{7}{|c|}{$\begin{array}{l}\text { ESQ (percent who guessed } \\
\text { receiving) }\end{array}$} \\
\hline Stimulant & $15 \%$ & $75 \%$ & $15 \%$ & $10 \%$ & $0 \%$ & $80 \%$ \\
\hline Sedative & $35 \%$ & $15 \%$ & $30 \%$ & $20 \%$ & $15 \%$ & $5 \%$ \\
\hline Cannabinoid & $20 \%$ & $5 \%$ & $10 \%$ & $10 \%$ & $15 \%$ & $10 \%$ \\
\hline Placebo & $25 \%$ & $5 \%$ & $45 \%$ & $60 \%$ & $70 \%$ & $5 \%$ \\
\hline
\end{tabular}

Abbreviations: DEQ, drug effects questionnaire; ESQ, end of study questionnaire; POMS, profile of mood states; VAS, visual analog scales. Mean (SEM) values are changes from pre-capsule to immediately before encoding (session I) and retrieval (session 2). Bold values indicate significant differences ( $p<0.05)$ between drug and placebo groups (t-test; see SOM for statistics). 
Table 3 Cued Recollection Data from Session 2 for Negative, Neutral, and Positive Images

\begin{tabular}{|c|c|c|c|c|c|c|c|c|c|}
\hline & \multicolumn{3}{|c|}{ Placebo } & \multicolumn{3}{|c|}{ MDMA at encoding } & \multicolumn{3}{|c|}{ MDMA at retrieval } \\
\hline & Negative & Neutral & Positive & Negative & Neutral & Positive & Negative & Neutral & Positive \\
\hline Hits & $0.7 \mid(0.04)$ & $0.60(0.04)$ & $0.62(0.05)$ & $0.69(0.03)$ & $0.59(0.03)$ & $0.63(0.03)$ & $0.72(0.03)$ & $0.60(0.04)$ & $0.63(0.03)$ \\
\hline FAs & $0.10(0.02)$ & $0.09(0.02)$ & $0.12(0.01)$ & $0.10(0.02)$ & $0.11(0.02)$ & $0.13(0.02)$ & $0.15(0.04)$ & $0.13(0.04)$ & $0.19(0.03)$ \\
\hline Accuracy & $0.61(0.04)$ & $0.51(0.04)$ & $0.50(0.04)$ & $0.59(0.03)$ & $0.48(0.03)$ & $0.49(0.03)$ & $0.58(0.04)$ & $0.47(0.04)$ & $0.45(0.03)$ \\
\hline Hi Conf Acc & $0.52(0.05)$ & $0.41(0.04)$ & $0.42(0.05)$ & $0.46(0.04)$ & $0.35(0.03)$ & $0.34(0.03)$ & $0.50(0.04)$ & $0.39(0.04)$ & $0.37(0.03)$ \\
\hline R Estimate & $0.40(0.05)$ & $0.28(0.05)$ & $0.31(0.04)$ & $0.25(0.05)$ & $0.25(0.03)$ & $0.21(0.04)$ & $0.33(0.05)$ & $0.26(0.06)$ & $0.27(0.04)$ \\
\hline F Estimate & $1.10(0.11)$ & $0.92(0.08)$ & $0.76(0.11)$ & $1.26(0.09)$ & $0.89(0.09)$ & $0.97(0.09)$ & $1.11(0.10)$ & $0.73(0.09)$ & $0.65(0.06)$ \\
\hline
\end{tabular}

Abbreviations: Acc, accuracy; F, familiarity; FA, false alarm; Hi Conf, high confidence; R, recollection. Values are means with SEM in parentheses except for the R and F estimates, which are point estimates of the aggregate ROC curves and standard deviations of the bootstrapping distributions.

Table 4 Recognition Data from Session 2 for Negative, Neutral, and Positive Images

\begin{tabular}{|c|c|c|c|c|c|c|c|c|c|}
\hline & \multicolumn{3}{|c|}{ Placebo } & \multicolumn{3}{|c|}{ MDMA at encoding } & \multicolumn{3}{|c|}{ MDMA at retrieval } \\
\hline & Negative & Neutral & Positive & Negative & Neutral & Positive & Negative & Neutral & Positive \\
\hline FAs & $0.05(0.01)$ & $0.05(0.01)$ & $0.07(0.01)$ & $0.05(0.01)$ & $0.07(0.01)$ & $0.07(0.02)$ & $0.09(0.03)$ & $0.08(0.03)$ & $0.12(0.03)$ \\
\hline Accuracy & $0.84(0.02)$ & $0.84(0.03)$ & $0.83(0.03)$ & $0.82(0.02)$ & $0.82(0.02)$ & $0.80(0.03)$ & $0.81(0.03)$ & $0.84(0.03)$ & $0.78(0.04)$ \\
\hline R Acc & $0.69(0.03)$ & $0.68(0.03)$ & $0.65(0.03)$ & $0.58(0.04)$ & $0.62(0.04)$ & $0.53(0.04)$ & $0.64(0.04)$ & $0.69(0.03)$ & $0.63(0.03)$ \\
\hline IRK F Hits & $0.66(0.06)$ & $0.66(0.07)$ & $0.70(0.07)$ & $0.66(0.04)$ & $0.73(0.04)$ & $0.75(0.05)$ & $0.70(0.05)$ & $0.74(0.05)$ & $0.72(0.05)$ \\
\hline IRK F FAs & $0.05(0.01)$ & $0.05(0.01)$ & $0.06(0.01)$ & $0.05(0.01)$ & $0.07(0.01)$ & $0.07(0.02)$ & $0.08(0.03)$ & $0.09(0.03)$ & $0.11(0.03)$ \\
\hline IRK F Acc & $0.61(0.06)$ & $0.62(0.07)$ & $0.63(0.07)$ & $0.61(0.05)$ & $0.66(0.04)$ & $0.67(0.05)$ & $0.62(0.05)$ & $0.66(0.06)$ & $0.61(0.06)$ \\
\hline
\end{tabular}

Abbreviations: Acc, accuracy; F, familiarity; FA, false alarm; Hi Conf, high confidence; IRK, independence remember/know; R, recollection. Values are means with SEM in parentheses.

underestimation. Familiarity accuracy is measured as

$\frac{p(\text { "know" } \mid \text { old })}{1-p(\text { "remember" } \mid \text { old })}-\frac{p(\text { "know" } \mid \text { new })}{1-p(\text { "remember" } \mid \text { new })}$

In order to avoid negative familiarity estimates and dividing by 0 , floor and ceiling hits and false alarms were replaced by $0.5 / N$ and $1-0.5 / N$, respectively, where $N$ is the maximum number of hits and false alarms that could be made (Macmillan and Creelman, 1991). Note that each of these estimates corrects for subjective responses to nonstudied items, thereby estimating recollection and familiarity unique to items studied in the encoding phase.

\section{Statistical Analysis}

Encoding and Retrieval groups were compared separately to the Placebo group. Cued recollection hits, false alarms, accuracy, and high confidence measures were submitted to 2 (group) $\times 3$ (valence) ANOVAs. Recognition hits, false alarms, accuracy, recollection estimates, and familiarity estimates were also submitted to $2 \times 3$ ANOVAs. When sphericity was violated, a Greenhouse-Geisser correction was applied to the degrees of freedom. Pairwise comparisons were conducted with $t$ tests.
Estimates of recollection and familiarity derived from ROC curves can be calculated individually for each participant (eg, Koen et al, 2013). However, because the number of studied and nonstudied items per condition was low (ie, 30 each compared to 60-150 in Koen et al), confidence data were collapsed across participants to generate aggregate ROC curves. Parameter reliability was assessed via non-parametric bootstrapping. For each condition, distributions of recollection and familiarity estimates were generated by randomly sampling 20 subjects with replacement and running an ROC analysis (10 000 iterations). Pairwise comparisons were made by subtracting distributions and calculating what proportion of the difference distribution lay above 0 . Confidence intervals for the difference of two means were obtained from the 2.5 and $97.5 \%$ quantiles of the difference distributions.

\section{RESULTS}

\section{Cued Recollection: Placebo vs Encoding}

Raw cued recollection performance was directly related to valence as indicated by main effects on hits $(\mathrm{F}(2,76)=14.109$, $\left.p<0.001, \quad \eta_{p}^{2}=0.271\right)$ and accuracy $(\mathrm{F}(2,76)=14.259$, 
Hit Rate

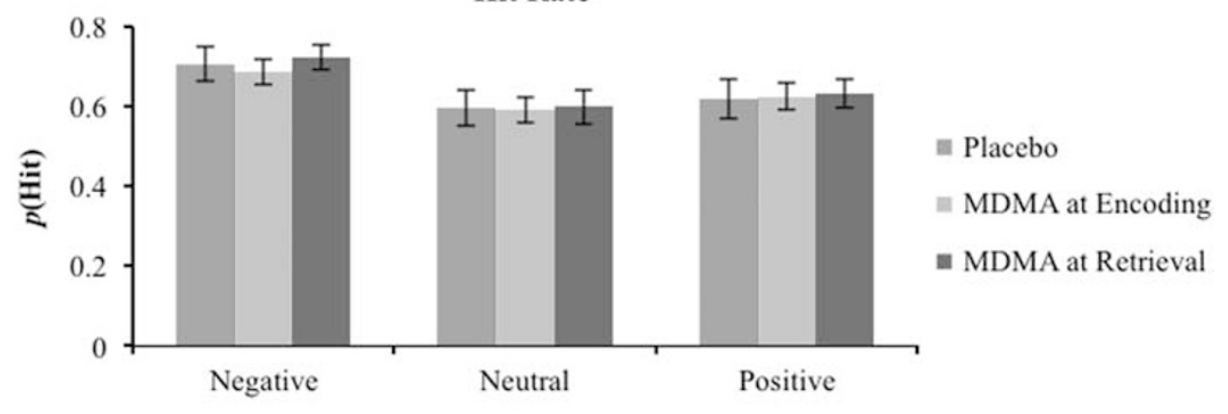

Cued Recollection:
False Alarm Rate

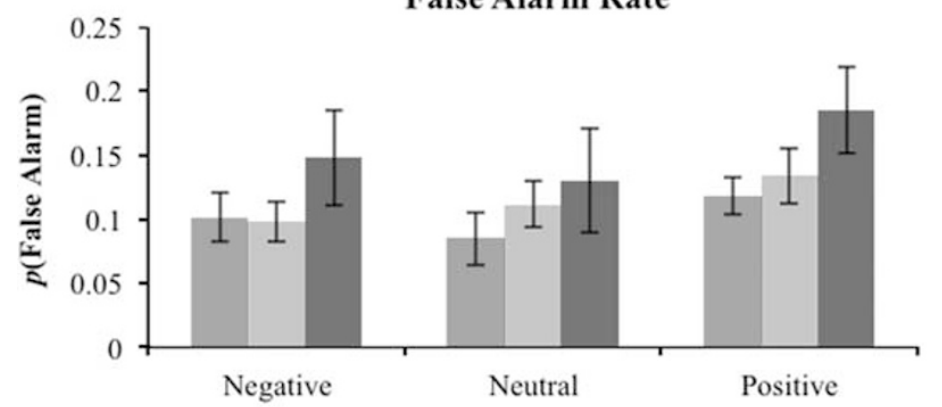

= Placebo

In MDA at Encoding

n MDMA at Retrieval

C

Cued Recollection:

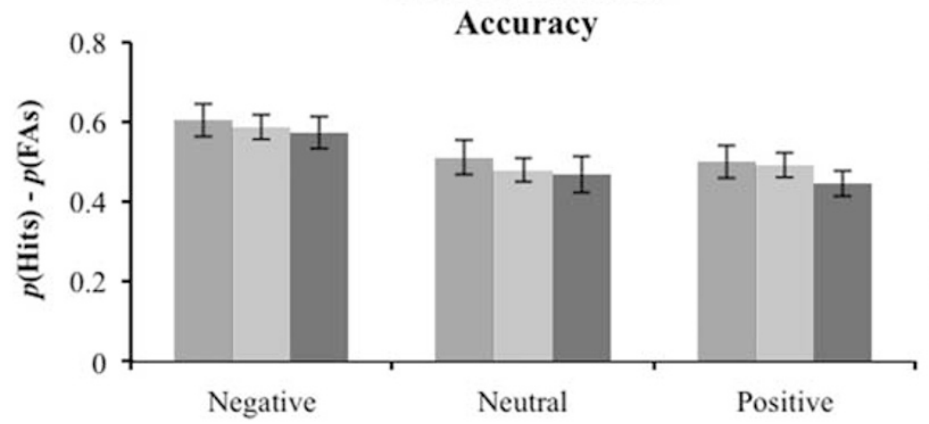

Placebo

= MDMA at Encoding

- MDMA at Retrieval

Figure I Raw performance on the cued recollection task. (a) Mean hit rates, (b) false alarm rates, and (c) accuracy (hit rates-false alarm rates). Error bars are SEM.

$\left.p<0.001, \eta_{p}^{2}=0.273\right)$. These main effects were due to the typical advantage for negative stimuli (Figure $1 \mathrm{a}$ and $\mathrm{c}$ ). There was a trending main effect of valence on false alarms $\left(\mathrm{F}(2,76)=2.488, p=0.090, \eta_{p}^{2}=0.061\right)$ due to more false alarms for positive stimuli (Figure $1 \mathrm{~b}$ ). No other main effects or interactions were found (all F's $<2.000$, all $p$ 's $>0.200$ ).

The distributions of DPSD-based recollection and familiarity estimates from the bootstrapping procedure were all normal (Figure 2). Negative (95\% CI: (0.004, 0.297), $p=0.022)$ and positive (95\% CI: $(-0.008,0.207), p=0.034)$ recollection estimates in the Encoding group were reduced compared with the Placebo group, though the confidence interval of the difference distribution for positive recollection estimates implied a less reliable effect. The Encoding and Placebo groups did not differ on neutral recollection estimates or familiarity estimates (all p's $>0.100$ ), though there was trend for greater positive familiarity estimates in the Encoding group (95\% CI: $(-0.085,0.465), p=0.078)$.

\section{Recognition: Placebo vs Encoding}

The ANOVAs on hits, false alarms, and accuracy from the picture recognition test were not significant (all F's $<1$ and $p$ 's $>0.250)$, except for a trending effect of valence on false alarms $\left(F(2,76)=2.286, p=0.109, \eta_{p}^{2}=0.057\right)$ due to greater false alarms to positive pictures.

The ANOVA on recollection estimates from the IRK procedure revealed a main effect of valence $(\mathrm{F}(2,76)=3.962$, $\left.p=0.007, \eta_{p}^{2}=0.122\right)$ and a marginal effect of group $\left(\mathrm{F}(1,38)=5.289, p=0.054, \eta_{p}^{2}=0.094\right)$ with no interaction $(\mathrm{F}(2,76)=1.559, p=0.217)$. The main effect of valence was due to smaller recollection estimates for positive pictures, and the main effect of group was due to attenuated recollection in the Encoding group (Figure 3). Although the interaction was not significant, exploratory contrasts found both negative $(t(38)=2.154, p=0.038, d=0.681)$ and positive $(t(38)=2.164, p=0.037, d=0.684)$ recollection estimates to be lower in the Encoding group compared with 
a

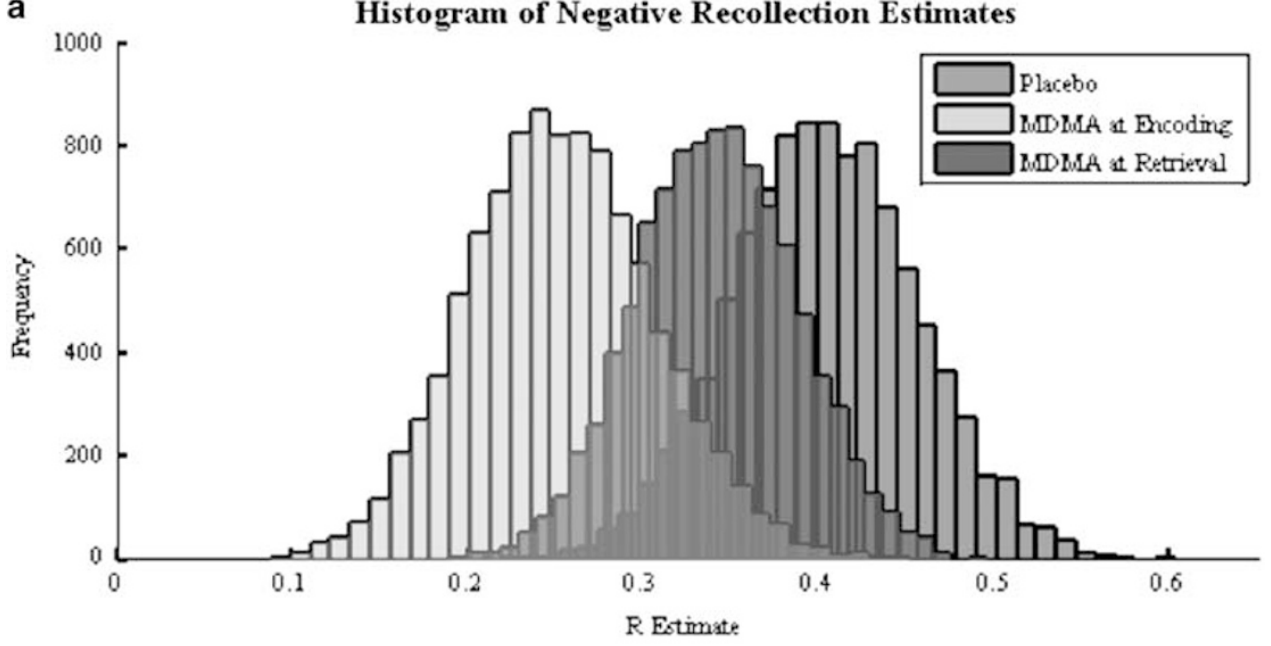

b

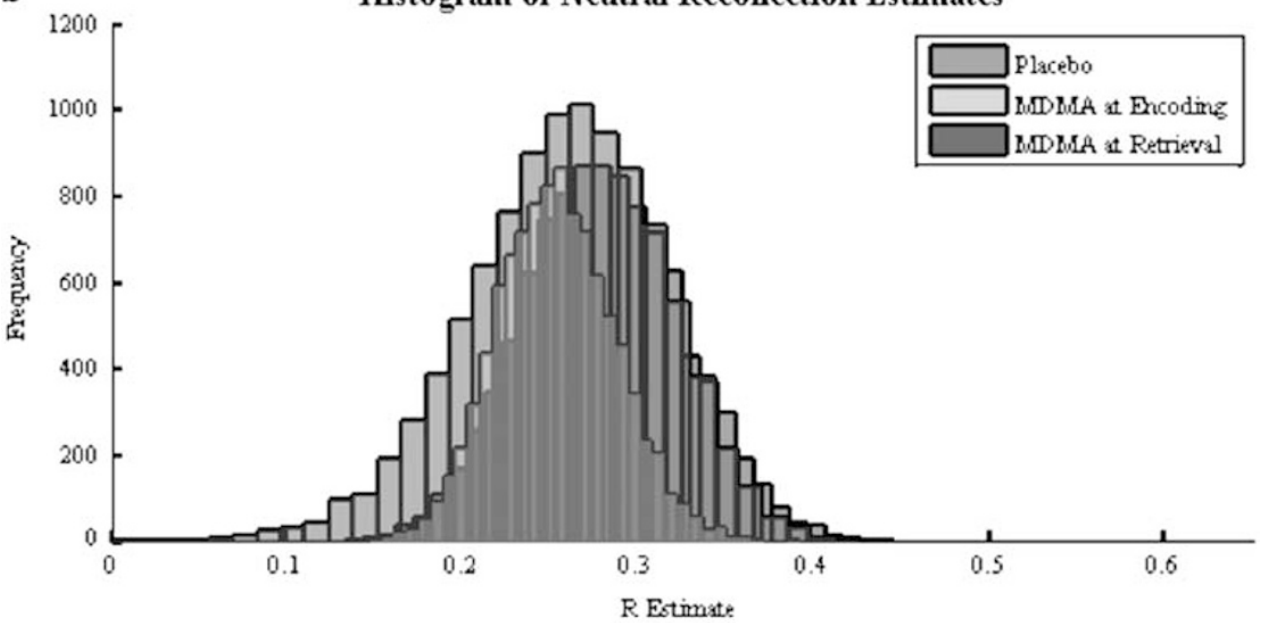

C

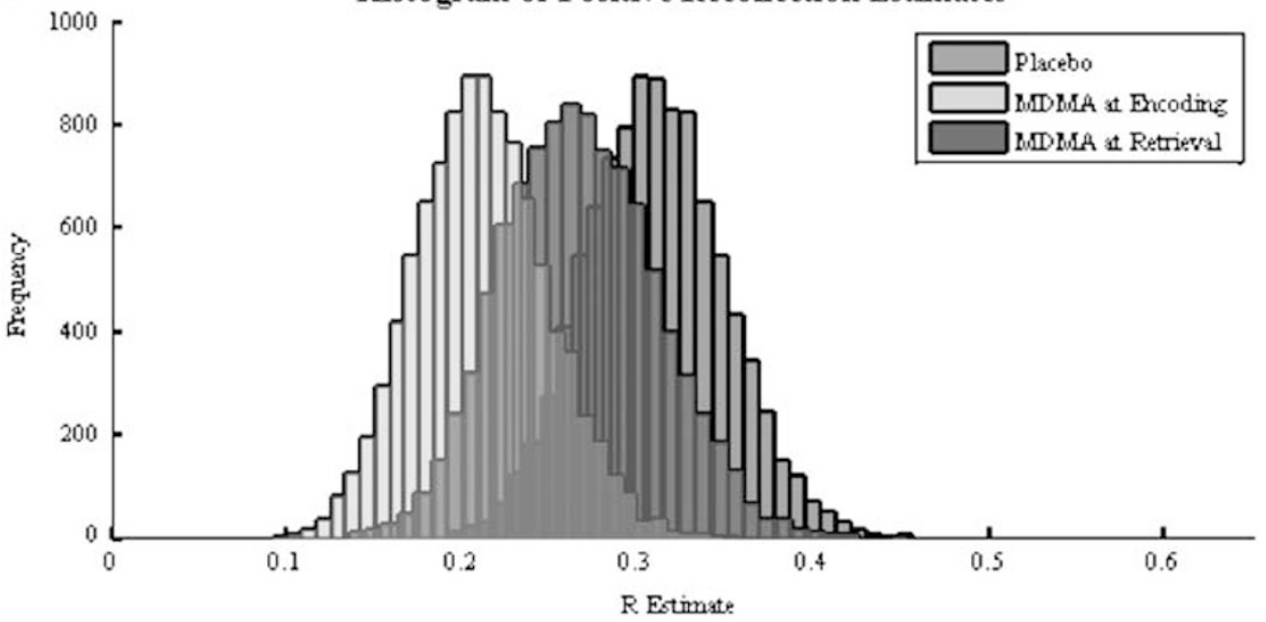

Figure 2 Distributions of (a) negative, (b) neutral, and (c) positive dual process signal detection-based recollection estimates generated from the bootstrapping procedure on the cued recollection confidence data.

the Placebo group with no reliable difference between neutral estimates $(t(38)=1.079, p=0.287, d=0.341)$, consistent with the DPSD-based recollection estimates. There were no main effects or interactions on familiarity estimates (all F's $<1$ and all $p$ 's $>0.250$ ).

\section{Cued Recollection: Placebo vs Retrieval}

Emotional valence strongly modulated hits $(\mathrm{F}(2,76)=18.143$, $\left.p<0.001, \quad \eta_{p}^{2}=0.323\right)$ and accuracy $\quad(\mathrm{F}(2,76)=16.417$, $\left.p<0.001, \eta_{p}^{2}=0.302\right)$ such that negative pictures showed a 

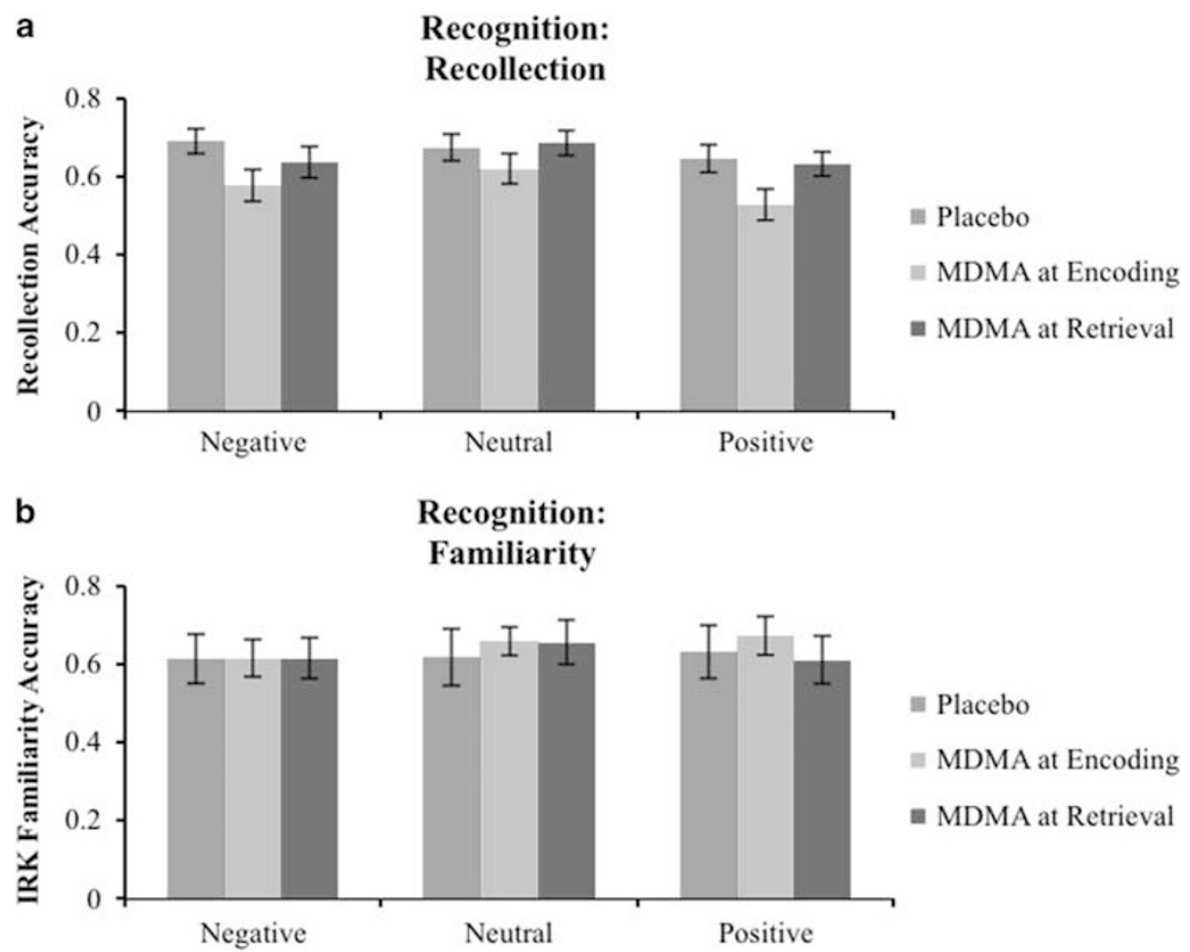

Figure 3 Mean independence remember/know estimates of (a) recollection and (b) familiarity from the recognition task. Error bars are SEM.

memory advantage. Valence modulated false alarms ( $\mathrm{F}$ $\left.(2,76)=4.121, p=0.020, \eta_{p}^{2}=0.098\right)$ due to more false alarms for positive stimuli. All other main effects and interactions were not statistically significant (all F's $<2.000, p>0.150$ ).

Although the difference between negative recollection estimates in the Placebo and Retrieval groups was not significant (95\% CI: $(-0.075,0.198), p=0.193)$, by comparison, the difference between the Encoding and Retrieval groups was also not significant (95\% CI: (-0.052, 0.224), $p=0.101)$. This can be seen in Figure $2 a$, which shows the negative recollection distribution of the Retrieval group lying in between those of the Encoding and Placebo groups. The distribution for positive recollection estimates of the Retrieval group was also in between the Placebo and Encoding groups with no difference between either of them (Figure 2c; Retrieval vs Placebo: 95\% CI: (-0.077, 0.157), $p=0.235$; Retrieval vs Encoding: 95\% CI: $(-0.124,0.147)$, $p=0.168$ ). There were no differences between the Retrieval and Placebo groups for neutral recollection estimates and familiarity estimates (all $p$ 's $>0.200$ ), though there was a trend for reduced neutral familiarity estimates in the Retrieval group (95\% CI: $(-0.057,0.421), p=0.066)$.

\section{Recognition: Placebo vs Retrieval}

The ANOVA on hits comparing Placebo and Retrieval groups did not reveal any main effects or interactions (all F's $<1$, all $p$ 's $>0.250$ ). However, the ANOVA on false alarms revealed a main effect of valence $(\mathrm{F}(2,76)=4.247, p=0.018$ $\left.\eta_{p}^{2}=0.101\right)$, again explained by greater false alarms for positive pictures. Although a main effect of group did not reach significance $\left(\mathrm{F}(1,38)=2.389, p=0.130, \eta_{p}^{2}=0.059\right)$, exploratory contrasts found that there was a trend for the
Retrieval group to false alarm more to positive stimuli than the Placebo group $(t(38)=1.816, p=0.077, d=0.075)$, consistent with the high confidence false alarms on the cued recollection test (SOM), and this was not found for other valences (all $t$ 's $<1.500$, all $p$ 's $>0.200$ ). Finally, there was a trending main effect of valence on accuracy $(\mathrm{F}(2,76)=2.695$, $\left.p=0.074, \eta_{p}^{2}=0.066\right)$ explained by decreased accuracy for positive pictures, owing to the increased false alarms. No other main effects and interactions were significant (all $F$ 's $<1.500$, all $p$ 's $>0.200)$.

There was a trending main effect of valence on recollection estimates $\left(F(2,76)=2.651, p=0.077, \eta_{p}^{2}=0.065\right)$ with positive recollection estimates being the smallest but no effect of group or interaction (all $F$ 's $<2.000$, all $p$ 's $>0.150$ ). Although there were no between-group differences, exploratory contrasts were conducted to determine consistent trends between the cued recollection and picture recognition tests, as were found among the Encoding group's analyses. These analyses found that both negative $(t(19)=2.146, p=0.045$, $d=0.480)$ and positive $(t(19)=2.393, p=0.027, d=0.535)$ recollection estimates were reduced compared to neutral estimates in the Retrieval group, but neither of these effects was found in the Placebo group (Figure 3a; neutral vs negative: $t(38)=0.566, \quad p=0.578$; neutral $v s$ positive: $t(19)=1.163, p=0.259)$. There were no main effects or interactions on the familiarity estimates (all F's $<1$ and all p's $>0.250)$.

\section{DISCUSSION}

We found that MDMA diminished the encoding of emotional information, both negative and positive, and this appeared to be specific to recollection, as there was no 
evidence for such an effect on familiarity. MDMA during retrieval tended to have subtler effects that mirrored those at encoding. These effects were found in recollection estimates produced from the DPSD analysis and the remember/know procedure, indicating that MDMA weakened episodic memory representations. Although prior work suggests amnestic effects of MDMA on the encoding of neutral items (Kuypers and Ramaekers, 2005), our study better isolated encoding and showed effects largest on emotional material.

Our finding that MDMA appeared to have a stronger effect on emotional memory is consistent with previous work demonstrating emotionally specific encoding effects of other drugs (eg, alcohol, Weafer et al, 2016; dextroamphetamine and $\Delta 9$-tetrahydrocannabinol, Ballard et al, 2013; ketamine, Becker et al, 2017). Alcohol and THC at encoding diminish both negative and positive memory, and dextroamphetamine at encoding enhances both negative and positive memory. These effects of dextroamphetamine are particularly interesting in light of our results with MDMA. In spite of their similar pharmacological profile and subjective effects, MDMA and dextroamphetamine have opposite effects on emotional memory, potentially because MDMA's defining effects on synaptic serotonin or $5-\mathrm{HT}_{2 \mathrm{~A}}$ receptor stimulation oppose its stimulant effects.

MDMA at retrieval had weak effects, but the Retrieval group's data did not completely mimic those of the Placebo group. For example, emotional recollection estimates from the cued recollection test were in between the Placebo and Encoding groups, suggesting slightly impaired retrieval of emotional information under MDMA. Another effect of MDMA on memory retrieval was a tendency to increase false alarms similar to dextroamphetamine (Ballard et al, 2014). The trends with MDMA on false alarms were most apparent for positive pictures, especially high confidence false alarms on the cued recollection test (SOM). Related to this finding, higher doses of MDMA (100 mg or $1.5 \mathrm{mg} / \mathrm{kg}$ ) have been shown to enhance visual imagery for positive autobiographical memories (Carhart-Harris et al, 2014) and processing fluency for emotionally positive words (Baggott et al, 2015). Therefore, MDMA during retrieval may have enhanced visual imagery and processing fluency of positive items, processes that recently have been shown to drive false recollections of pictures in other work (Doss et al, 2016).

Returning to the significant encoding effects, whereas MDMA-induced decreases in the recollection of negative information speak to its potential role as an adjunct to psychotherapy, the amnestic effects on positive recollection may interfere with its therapeutic value. There is a high comorbidity for depression in PTSD (Campbell et al, 2007), and depression has been associated with a deficit in memories for positive experiences (Dillon, 2015). Therefore, caution may be warranted when considering MDMAassisted psychotherapy for PTSD patients with comorbid depression.

A notable observation in this study was MDMA did not affect raw proportions of hits or memory accuracy on the cued recollection test, which was designed to objectively assess the recollection of pictures. However, our measures of recollection based on the DPSD model and IRK procedure indicated that MDMA significantly impacted the recollection of emotional memories. These different patterns may relate to the distinction between the number of events that can be successfully recollected, on the one hand, and the amount of vividness or precision of recollected details associated with these retrieved events on the other hand (eg, quantity $v s$ quality, Scimeca et al, 2011; success $v s$ precision, Harlow and Yonelinas, 2016). In this light, our findings suggest that MDMA affected the recollection of details associated with emotional events but not necessarily the overall ability to recollect the occurrence of an emotional event.

The idea that MDMA alters the recollection of details associated with emotional events but not memory for the occurrence of the event may have important clinical implications. To the extent that memory retrieval is required to trigger reconsolidation (Kroes et al, 2016) and the encoding of new information is required to alter a memory trace (Sevenster et al, 2013), a strong amnestic effect may not be desirable for a therapeutic agent. PTSD is associated with memory impairments (Johnsen and Asbjørnsen, 2008), so any additional amnestic effect that precludes successful encoding and retrieval could actually hinder psychotherapy. Nevertheless, abolishing the precise details of traumatic memories, weakening their associations, or re-encoding these memories with novel, less emotional associations may be advantageous for preventing generalizations of fear to innocuous stimuli (ie, second-order conditioning, Wessa and Flor, 2007). Such effects could also prevent the incorporation of trauma-related information when thinking about the future (Brown et al, 2013). Future work should explore the precise modifications MDMA has on memory, whether higher doses may be more effective at producing such modifications, and how these modifications compare to other drug-induced memory distortions.

\section{FUNDING AND DISCLOSURE}

HdW has received a GRAND research award from Pfizer, donation of a study drug from Indivior, support for a research study from Insys Therapeutics, and consulting fees from Bristol-Myers-Squibb, Jazz Pharmaceuticals, Marinus, and Organon. None of these were related to the research presented here. This project was supported by DA031796 and DA02812. The remaining authors have no conflict of interest.

\section{ACKNOWLEDGMENTS}

We would like to thank Joshua Koen and Omid Kardan for important conversations regarding the bootstrapping analysis.

\section{REFERENCES}

Baggott MJ, Kirkpatrick MG, Bedi G, de Wit H (2015). Intimate insight: MDMA changes how people talk about significant others. J Psychopharmacol 29: 669-677.

Ballard ME, Gallo DA, de Wit H (2013). Pre-encoding administration of amphetamine or THC preferentially modulates emotional memory in humans. Psychopharmacology 226: 515-529.

Ballard ME, Gallo DA, de Wit H (2014). Amphetamine increases errors during episodic memory retrieval. J Clin Psychopharmacol 34: 85-92.

Becker B, Steffens M, Zhao Z, Kendrick KM, Neumann C, Weber B et al (2017). General and emotion-specific neural effects of 
ketamine during emotional memory formation. Neuroimage 150: 308-317.

Bedi G, Phan KL, Angstadt M, de Wit H (2009). Effects of MDMA on sociability and neural response to social threat and social reward. Psychopharmacology 207: 73-83.

Bershad AK, Miller MA, Baggott MJ, de Wit H (2016). The effects of MDMA on socio-emotional processing: does MDMA differ from other stimulants? J Psychopharmacol 30: 1248-1258.

Brohawn KH, Offringa R, Pfaff DL, Hughes KC, Shin LM (2010). The neural correlates of emotional memory in posttraumatic stress disorder. Biol Psychiatry 68: 1023-1030.

Brown AD, Root JC, Romano TA, Chang LJ, Bryant RA, Hirst W (2013). Overgeneralized autobiographical memory and future thinking in combat veterans with posttraumatic stress disorder. J Behav Ther Exp Psychiatry 44: 129-134.

Campbell DG, Felker BL, Liu CF, Yano EM, Kirchner JE, Chan D et al (2007). Prevalence of depression-PTSD comorbidity: implications for clinical practice guidelines and primary carebased interventions. J Gen Intern Med 22: 711-718.

Carhart-Harris RL, Wall MB, Erritzoe D, Kaelen M, Ferguson B, de Meer I et al (2014). The effect of acutely administered MDMA on subjective and BOLD-fMRI responses to favourite and worst autobiographical memories. Int $J$ Neuropsychopharmacol 17: 527-540.

Carmack SA, Wood SC, Anagnostaras SG (2010). Amphetamine and extinction of cued fear. Neurosci Lett 468: 18-22.

Dillon DG (2015). The neuroscience of positive memory deficits in depression. Front Psychol 6: 1-12.

Doss MK, Bluestone MR, Gallo DA (2016). Two mechanisms for constructive recollection: perceptual recombination and conceptual fluency. J Exp Psychol Lear Mem Cogn 42: 1747-1758.

Folstein MF, Luria R (1973). Reliability, validity, and clinical application of the Visual Analogue Mood Scale. Psychol Med 3: 479-486.

Harlow IM, Yonelinas AP (2016). Distinguishing between the success and precision of recollection. Memory 24: 114-127.

Hysek CM, Schmid Y, Simmler LD, Domes G, Heinrichs M, Eisenegger $C$ et al (2014). MDMA enhances emotional empathy and prosocial behavior. Soc Cogn Affect Neurosci 9: 1645-1652.

Johnsen GE, Asbjørnsen AE (2008). Consistent impaired verbal memory in PTSD: a meta-analysis. J Affect Disord 111: 74-82.

Koen JD, Aly M, Wang W-C, Yonelinas AP (2013). Examining the causes of memory strength variability: recollection, attention failure, or encoding variability? J Exp Psychol Learn Mem Cogn 39: $1726-1741$.

Koen JD, Barrett FS, Harlow IM, Yonelinas AP (2016). The ROC Toolbox: a toolbox for analyzing receiver-operating characteristics derived from confidence ratings. Behav Res Methods Advance online publication 49: 1399-1406.

Kroes MC, Schiller D, LeDoux JE, Phelps EA (2016). Translational approaches targeting reconsolidation. Curr Top Behav Neurosci 28: 197-230.

Kuypers KP, Ramaekers JG (2005). Transient memory impairment after acute dose of $75 \mathrm{mg}$ 3.4-methylene-dioxymethamphetamine. J Psychopharmacol 19: 633-639.
Lang PJ, Bradley MM, Cuthbert BN (2008). International Affective Picture System (IAPS): Affective Ratings of Pictures and Instruction Manual. Technical Report A-8. University of Florida: Gainesville, FL.

Macmillan NA, Creelman CD (1991). Detection Theory: A User's Guide. Cambridge University Press: New York, NY.

Mather M, Clewett D, Sakaki M, Harley CW (2016). Norepinephrine ignites local hot spots of neuronal excitation: How arousal amplifies selectivity in perception and memory. Behav Brain Sci 39: e200.

McNair D, Lorr M, Droppelman LF (eds). Manual for the Profile of Mood States. Educational and Industrial Testing Service: San Diego, 1971.

Morean ME, de Wit H, King AC, Sofuoglu M, Rueger SY, O’Malley SS (2013). The Drug Effects Questionnaire: psychometric support across three drug types. Psychopharmacology 227: 177-192.

Phelps EA, Sharot T (2008). How (and why) emotion enhances the subjective sense of recollection. Curr Dir Psychol Sci 17: 147-152.

Rauch SL, Shin LM, Phelps EA (2006). Neurocircuitry models of posttraumatic stress disorder and extinction: human neuroimaging research-past, present, and future. Biol Psychiatry 60: 376-382.

Rubin DC, Berntsen D, Bohni MK (2008). A memory-based model of posttraumatic stress disorder: evaluating basic assumptions underlying the PTSD diagnosis. Psychol Rev 115: 985-1011.

Scimeca JM, McDonough IM, Gallo DA (2011). Quality trumps quantity at reducing memory errors: implications for retrieval monitoring and mirror effects. J Mem Lang 65: 363-377.

Sessa B (2016). MDMA and PTSD treatment: 'PTSD: from novel pathophysiology to innovative therapeutics'. Neurosci Lett 649: 176-180.

Sevenster D, Beckers T, Kindt M (2013). Prediction error governs pharmacologically induced amnesia for learned fear. Science 339: 830-833.

Smith ME (2005). Bilateral hippocampal volume reduction in adults with post-traumatic stress disorder: a meta-analysis of structural MRI studies. Hippocampus 15: 798-807.

van Wel JH, Kuypers KP, Theunissen EL, Bosker WM, Bakker K, Ramaekers JG (2011). Blockade of 5-HT2 receptor selectively prevents MDMA-induced verbal memory impairment. Neuropsychopharmacology 36: 1932-1939.

Weafer J, Gallo DA, de Wit H (2016). Acute effects of alcohol on encoding and consolidation of memory for emotional stimuli. J Stud Alcohol Drugs 77: 86-94.

Wessa M, Flor H (2007). Failure of extinction of fear responses in posttraumatic stress disorder: evidence from second-order conditioning. Am J Psychiatry 164: 1684-1692.

White TL, Justice AJ, de Wit H (2002). Differential subjective effects of D-amphetamine by gender, hormone levels and menstrual cycle phase. Pharmacol Biochem Behav 73: 729-741.

Yonelinas AP (2002). The nature of recollection and familiarity: a review of 30 years of research. J Mem Lang 46: 441-517.

Young MB, Andero R, Ressler KJ, Howell LL (2015). 3,4Methylenedioxymethamphetamine facilitates fear extinction learning. Transl Psychiatry e634.

Supplementary Information accompanies the paper on the Neuropsychopharmacology website (http://www.nature.com/npp) 